\section{Acaricides utilisables dans la lutte contre les tiques aux Antilles : résultats d'essais en Guadeloupe}

\author{
N. Barré ${ }^{1}$ \\ M. Fargetton ${ }^{2}$ \\ R. Aprelon ${ }^{1}$ \\ L. Coulibando ${ }^{1}$
}

BARRE N., FARGETTON M., APRELON R., COULIBANDO L. Acaricides utilisables dans la lutte contre les tiques aux Antilles: résul tats d'essais en Guadeloupe. Revue Elev. Méd. vét. Pays trop., 1995, 48 (4) : $351-356$

Un acaricide pyréthrinoïde, la deltaméthrine (Butox $50^{\text {wis) }}$, une amidine,

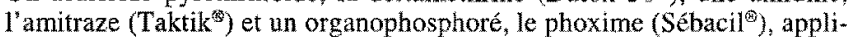
cables en aspersion, ainsi que deux pyréthrinoïdes administrables en dépôt dorsal ("pour-on") ou en taches ("spot-on"), la fluméthrine (Bayti$\mathrm{col}^{\text {s }}$ "pour-on") et la deltamethrine (formulation "pour-on"), ont été testés en Guadeloupe sur des bovins au pâturage naturellement infestés par des adultes de la tique Amblyomma variegatum. Pour les produits administrables en aspersion, la meilleure efficacité est obtenue avec l'amitraze (98,5 p. 100 de réduction de l'infestation). L'efficacité est encore meilleure avec les deux pyréthrinoïdes testés en "pour-on" $(99.3$ p. 100 de réduction avec la fluméthrine), ou en "spot-on" (100 p. 100 de réduction avec la fluméthrine et la deltaméthrine). Outre son efficacité, la technique du "spot-on" est la mieux adaptée au contexte de l'élevage à l'attache pratiqué aux Antilles. Ce mode de traitement est 2 à 3 fois plus rapide que l'aspersion permettant, malgré le coût élevé des produits, un gain en temps, done en personnel qui peut rendre cette méthode de détiquage compétitive, en particulier dans des perspectives d'éradication des tiques.

Mots-clês : Bovin - Tique - Amblyorma - Boophilus - Haematobia Maladies transmissibles par tiques - Lutte antiacarien - Acaricide - Pyréthrine de synthèse - Deltaméthrine - Amitraze - Phoxime - Elevage Guadeloupe - Caraíbes.

\section{CIRAD-EMVT, BP 515, 97165 Pointe-à-Pitre, Guadeloupe, France}

2. INRA-CRAAG, BP 515, 97165 Pointe-à-Pitre, Guadeloupe, France

Reçu le 11.9.95, accepté le 18.3.96.

\section{Introduction}

Deux espèces de tiques, Amblyomma variegatum (F., 1794) et Boophilus microplus (Canestrini, 1883), parasitent le bétail aux Antilles. La première est vectrice de la cowdriose en Guadeloupe, à Marie-Galante et à Antigue, la seconde des babésioses et de l'anaplasmose dans toutes les îles. De plus, $A$. variegatum est étroitement associé à la dermatophilose, qui se manifeste sous sa forme clinique sévère chez les races sensibles dans toutes les îles ou parties d'îles infestées par cette tique. La gravité de cette maladie et l'importance des pertes économiques qui lui sont liées justifient la mise en oeuvre de mesures de lutte dirigées spécifiquement contre elle, mais aussi contre Boophilus.

Deux stratégies de lutte contre les tiques sont envisageables : 1) diminution de l'infestation pour maintenir le parasitisme à un niveau compatible avec des performances de production des animaux satisfaisantes; 2) éradication complète et définitive des tiques d'une île, d'un groupe d'îles ou d'une région.

La première stratégie est appliquée depuis une trentaine d'années dans les Antilles françaises. Elle satisfait les éleveurs de bétail créole, notamment les éleveurs de bovins créoles hautement résistants aux maladies transmises par les tiques ou qui leurs sont associées. Elle n'empêche cependant pas les accidents sur les caprins, très sensibles à la cowdriose, ni sur les bovins croisés qui paient un lourd tribut à toutes les maladies liées aux tiques. Plus préoccupant, cette stratégie ne permet pas de limiter efficacement la propagation de la tique Amblyomma variegatum dans la Caraïbe. Depuis 1967. elle a colonisé 14 îles de la région et elle menace de gagner le continent. Afin de limiter ce risque, un programme d'éradication de cette espèce de tique dans les Petites Antilles, inspiré de celui déjà conduit à Porto-Rico (6), a été décidé (5). Ses objectifs, son état d'avancement et les contraintes ont été décrits $(2,4)$.

La mise en œuvre de ce programme basé sur l'application régulière, tous les 14 jours et pendant 2 ans au moins, d'acaricides sur le bétail (2), nécessite de disposer d'acaricides les moins toxiques possible, laissant peu ou pas de résidus, faciles et rapides d'emploi, hautement efficaces et si possible doués d'une action rémanente. De plus, des considérations financières ne seront pas étrangères au choix des produits. Après avoir réalisé 
quelques tests de sensibilité in vitro sur des souches d'Amblyomma variegatum de Guadeloupe (7), il est apparu utile de mesurer in vivo l'efficacité de divers acaricides et la facilité d'emploi de divers modes d'administration, étant entendu que l'élevage traditionnel aux Antilles comporte en grande majorité des cheptels de petite taille ( 6 à 8 bovins par exploitation), élevés à l'attache (au piquet), disséminés dans les campagnes, n'autorisant pas les équipements lourds du type bain détiqueur.

\section{Matériel et Méthodes}

\section{Tests d'efficacité}

Animaux expérimentaux, rythme des traitements, dénombrements des tiques

Les essais ont été conduits à la station expérimentale INRA du domaine de Gardel (Le Moule), dans une des principales zones d'élevage de la Guadeloupe, où les conditions bioclimatiques sont hautement favorables au développement de la tique Amblyomma variegatum $\left(24,6^{\circ} \mathrm{C}\right.$ en février à $27,3^{\circ} \mathrm{C}$ en août ; $1200 \mathrm{~mm}$ de pluies annuelles). Cinq lots de 13 à 20 vaches créoles chacun, conduites sur pâturage irrigué à Pangola, Digitaria decumbens (1 lot) ou sur pâturage naturel non irrigué à "petit-foin", Dichantium caricosum (les 4 autres lots) ont été utilisés.

Trois séries d'essais ont eu lieu en juin et en novembre 1994, et en avril 1995. Pendant trois mois au moins avant chaque essai, les animaux ne recevaient aucun traitement. Pour chaque acaricide utilisé, les traitements étaient effectués trois fois à 14 jours d'intervalle et les tiques adultes (mâles, femelles non gorgées, semi gorgées et gorgées) étaient dénombrées sur tout le corps de chaque animal, rentré dans un couloir de contention, à
$\mathrm{JO}, \mathrm{J} 3$ et $\mathrm{J} 7$ après chacun des traitements. De plus, une appréciation semi-quantitative de l'abondance des Boophilus et des mouches (Haematobia) était effectuée. Pour chacune de ces trois séries, un lot témoin n'était pas détiqué et l'infestation de ces animaux était suivie simultanément à celle des lots traités.

\section{Mesure de l'efficacité des traitements}

Elle pouvait être mesurée par le test d'Abott (1), qui corrige la mortalité constatée par celle éventuellement observée dans le lot témoin. Ce test ne peut s'appliquer rigoureusement que si le lot témoin est élevé dans des conditions identiques au lot traité, ce que les conditions expérimentales de cette étude n'ont pas permis. En effet, les lots étaient chacun sur des parcelles différentes de niveaux d'infestation différents et ceci pour éviter le passage du produit par contact des animaux détiqués vers les non détiqués s'ils avaient été sur le même pâturage (9).

Considérant le court délai entre le premier et le dernier dénombrement (35 à 38 jours), insuffisant pour qu'apparaissent des fluctuations naturelles très importantes des effectifs de tiques sur les animaux, on pouvait aussi apprécier l'efficacité en calculant la diminution de l'infestation dans chaque lot induite par le dernier traitement, par rapport à l'infestation initiale. Pour des produits ayant une certaine rémanence, la concentration optimale au niveau du poil peut être obtenue après plusieurs traitements, ce qui se passe d'ailleurs dans le cas d'une campagne de détiquage à intervalles réguliers. La réduction de l'infestation a ainsi été mesurée entre J0 et quelques jours après le troisième traitement. Ce choix va privilégier les produits à la fois actifs et rémanents.

\section{Produits acaricides utilisés (tableau I)}

Leurs dénominations et caractéristiques sont indiquées dans le tableau I. Les concentrations utilisées sont celles

TABLEAU I

Caractéristiques des acaricides utilisés

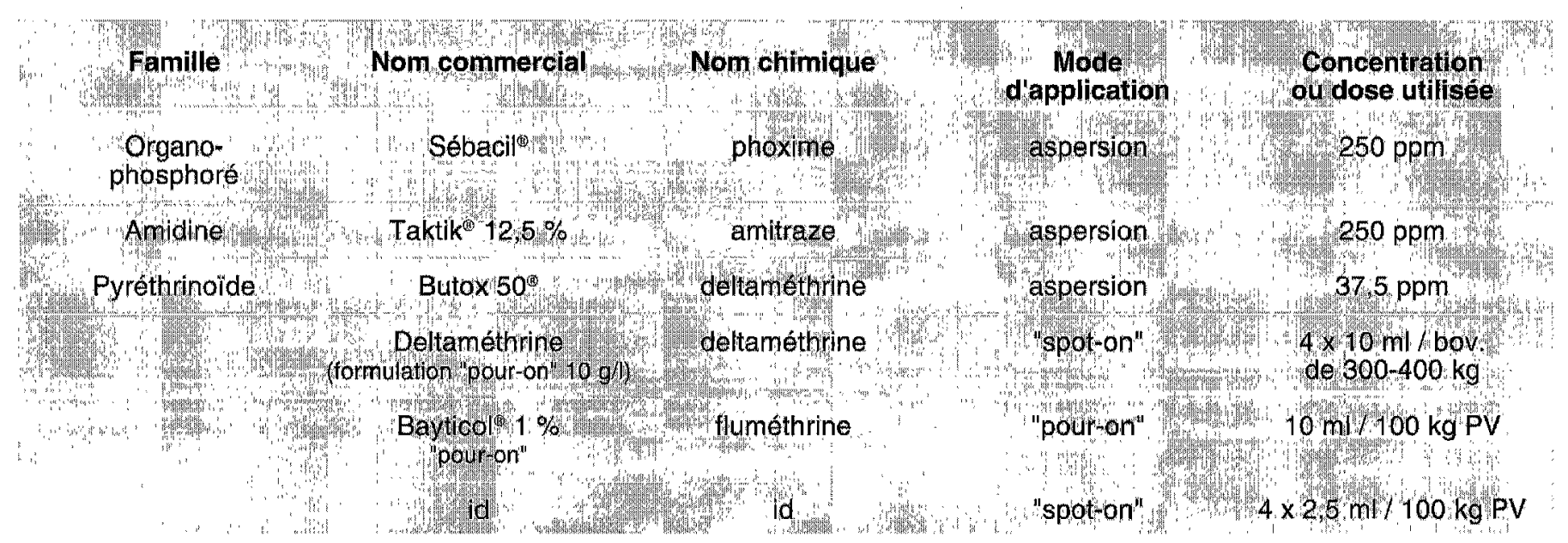


indiquées par le fabricant sur l'étiquette du flacon. Les produits en aspersion étaient administrés à l'aide d'un pulvérisateur à moteur à raison de $5 \mathrm{I}$ de solution prête à l'emploi par bovin. L'administration de la deltaméthrine et de la fluméthrine en "spot-on" ne figure pas dans les directives des fabricants, mais ceux-ci ont accepté que l'on teste ces produits selon ce mode d'application qui s'était avéré intéressant dans des expérimentations préliminaires (3). Il consiste à propulser à distance à l'aide d'un pistolet doseur (The Protector ${ }^{\circledR}$, Vetmark Services Limited, Cambridge), la dose fractionnée en quatre parties sur le haut des cuisses et des épaules, de chaque côté de l'animal.

\section{Facilité d'emploi de divers modes d'application}

L'application du produit en "pour-on" est peu adaptée aux conditions d'élevage aux Antilles, la grande majorité des animaux étant tenus au piquet, attachés par une chaîne et donc mobiles autour du point d'attache. Dans ces conditions, il est difficile de s'approcher suffisamment près des animaux pour déposer la dose de produit. L'application de l'acaricide en "spot-on" a donc été testée, comme cela a été décrit précédemment, et on a comparé la rapidité d'exécution avec le traitement classique en aspersion, et ceci chez 46 éleveurs totalisant 383 bovins élevés selon les méthodes traditionnelles.

\section{Résultats}

\section{Efficacité des acaricides sur Amblyomma variegatum}

Quelle que soit la période de l'année où ont eu lieu les essais : milieu de la saison sèche (avril), fin de la saison sèche (juin), ou fin des pluies (novembre), des tiques adultes dont des femelles, sont présentes sur les animaux, témoignant d'une activité de cette stase toute l'année dans les conditions de la Guadeloupe.

Les niveaux d'infestation en début d'essai sont très variables d'un lot à l'autre (moyenne de 2,72 à 27,9 tiques adultes par bovin, tableaux II et III) reflétant généralement des situations écologiques et un historique de lutte contre les tiques (ancienneté, intensité, régularité, etc.) différents.

Au cours des essais, les effectifs de tiques sur les animaux témoins non détiqués ont tous augmenté. Pendant les 35 à 38 jours de suivi, cette augmentation a été de 23 p. 100 (lot témoin 1, tableau III), à 68 p. 100 (lot témoin 2, tableau III). L'évolution naturelle de l'infestation va donc vers un accroissement du nombre de tiques adultes sur les animaux, la période d'observation correspondant à une phase dynamique de réinfestation des animaux, précédemment détiqués trois mois avant le début des essais.

TABleaU II

Nombre moyen d'Amblyomma variegatum adultes sur les animaux du lot témoin et sur ceux détiqués avec deux acaricides pyréthrinoïdes : la deltaméthrine (formulation "pour-on") (DEL) et le Bayticol "pour-on" (Bi , (BAY.POUR) appliqués en dépôt dorsal ou en taches (BAY.SPOT) sur les animaux

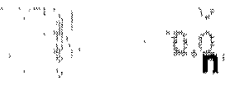

TEMOIN 1
Jo

(T1)

J3

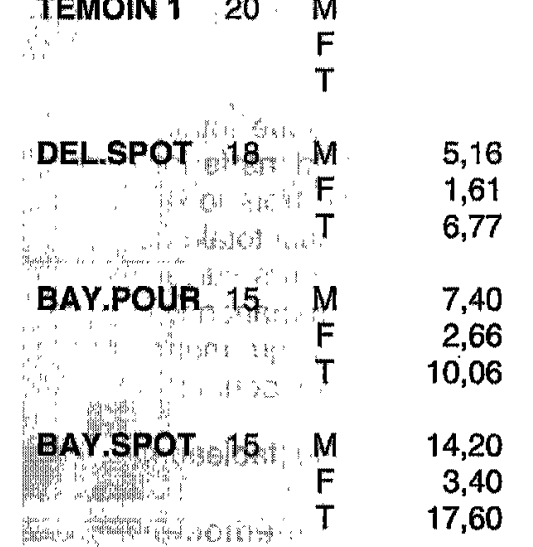

3,40

0,30

3,70

1,55

0,17

1,72

0,27

0,05

0,32

0,33

0

0,33

\section{0,07}

0,07

$\begin{array}{ll}0 & 0,07 \\ 0 & 0 \\ 0 & 0,07\end{array}$

\section{J14}

(T2)

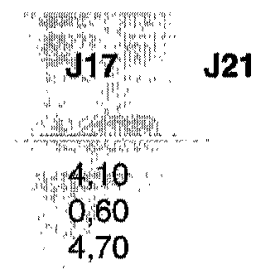

0,05

0,05

0,13

0

0,13

0,13

0

0,13

0
0
0
0
0
0
0
0
0

0
0
0
0,13
0
0,13

0,07
0
0,07

J28

(T3)

$\mathbf{J 3 1}$
$\mathbf{4}$
4,05
0,50
4,55

0,11
0
0,11

J35 a

$\%$ J38 Rou A

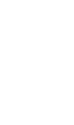

0,22

0

0,22

0,11

0,67

0,07

0,74

0

0

0,67

0

0,07
0

0,07

$M=$ mâles, $F=$ femelles, $T=$ mâles + femelles. Début de l'essai, 14 juin 1994, $n$; nombre de vaches par lot ; T1, T2, T3: traitements $1,2,3$;

$\% \mathrm{R}$ ou $\mathrm{A}$ : pourcentage de réduction ou d'augmentation de l'infestation. 
TABLEAU III

Nombre moyen d'Amblyomma variegatum adultes sur les animaux des lots témoins et sur ceux détiqués par aspersions au Butox $50^{\oplus}$ (début du 1er essai, 14 juin 1994 ; début du $2^{\circ}, 21$ avril 1995), au Sébacil ${ }^{\circledR}$ ( 21 avril) et au Taktik ${ }^{\circledR}(15$ novembre 1994)

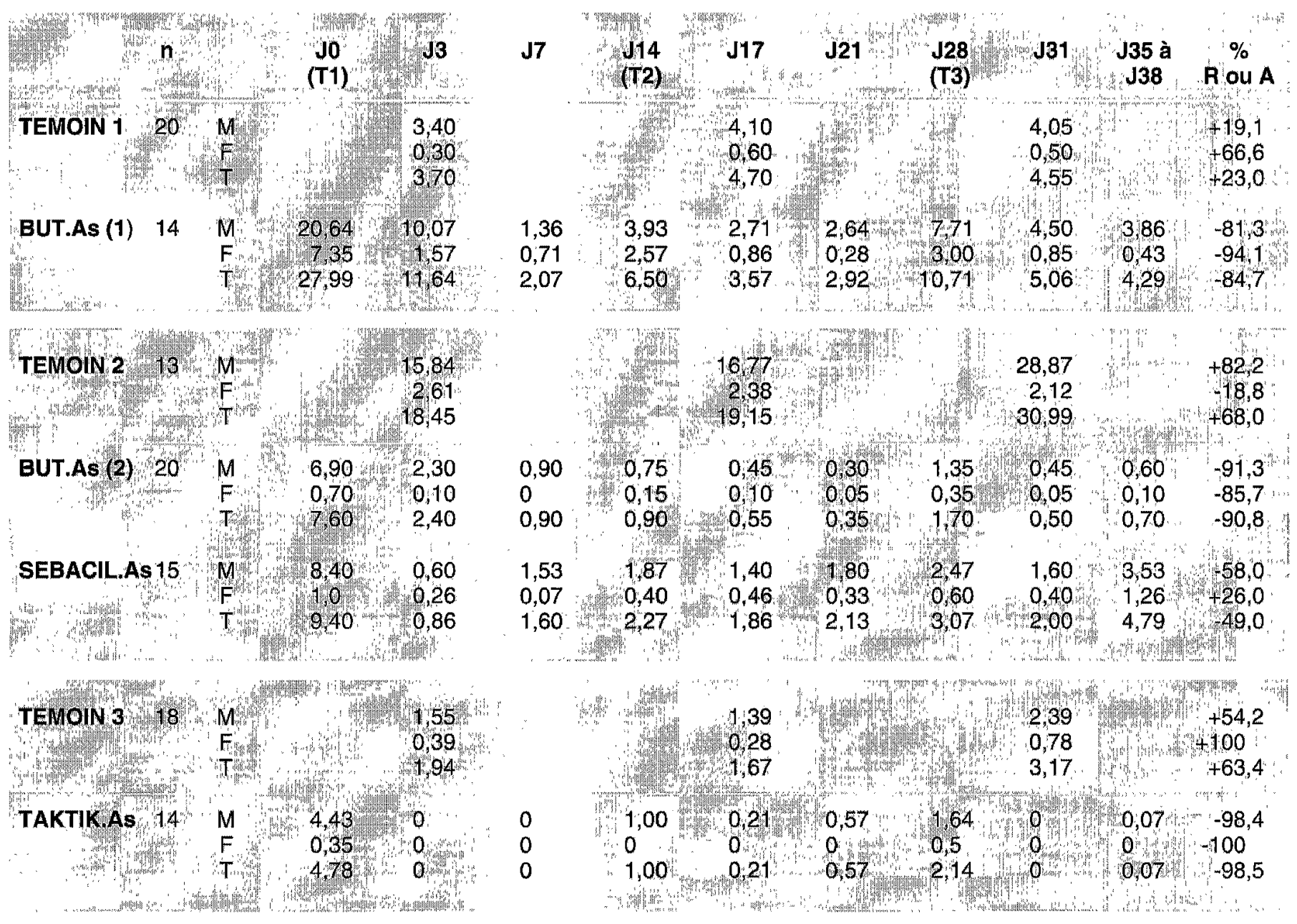

$\mathrm{n}$ : nombre de vaches par lot ; T1, T2, T3 : traitements 1,2 et $3 ; \%$ R ou $\mathrm{A}$ : pourcentage de réduction ou d'augrnentation de l'infestation.

Au contraire, le niveau d'infestation a fortement diminué dans les neuf lots traités, mais dans des proportions différentes en fonction des produits, et avec de fortes variations de cinétique de réinfestation.

Pyréthrinoïdes appliqués en dépôt dorsal ("pour-on") ou en taches ("spot-on") (tableau H)

La réduction obtenue dès le premier traitement est intense et rapide, surtout avec le Bayticol ${ }^{\oplus}$ qui ne laisse pratiquement plus de tiques vivantes au contrôle effectué 3 jours après traitement. Un résultat identique mais légèrement différé (entre 3 et 7 jours) est observé avec la deltaméthrine en formulation "pour-on". Huit jours après le troi- sième traitement, les tiques ont presque totalement disparu ( 1 seul mâle vivant dénombré dans le lot traité au Bayticol "pour-on" à J35) dans les trois lots traités en "pour-on" ou "spot-on". L'absence quasi totale de réinfestation par les femelles dans les 14 jours suivant chaque application confirme l'efficacité et la rémanence dés pyréthrinoïdes sur les mâles Amblyomma qui meurent avant de produire les phéromones attractives pour les femelles.

\section{Autres acaricides utilisés en aspersion (tableau III)}

La réduction de l'infestation est médiocre avec le Sébacil $^{\Theta}$ à la concentration utilisée puisqu'elle ne touche que la moitié des tiques. Elle est bonne, comprise entre 85 et 91 p. 100 de l'infestation initiale avec le Butox $50^{(}$. 
La meilleure efficacité $(98,5$ p. 100 de réduction) est obtenue avec le Taktik ${ }^{(3)}$ le seul produit utilisable en aspersion qui ait une efficacité totale sur les femelles après trois traitements.

\section{Efficacité sur les autres arthropodes parasites}

\section{Boophilus microplus}

Un seul lot était infesté par quelques Boophilus en début d'essai. II s'agit d'un lot traité au Butox $50^{\oplus}$. Plus aucun Boophilus n'a été retrouvé après le premier traitement.

\section{Haematobia irritans}

Tous les lots étaient fortement infestés, en début d'essai, par ce petit diptère hématophage, resté abondant dans tous les lots témoins pendant la durée de l'expérimentation. Par contre, cette mouche a immédiatement et totalement disparu des animaux dans les cinq lots traités avec un pyréthrinoïde. Elle a fortement diminué, sans disparaître complètement, dans le lot Sébaci ${ }^{\circledR}$ et le lot Taktik ${ }^{\circledR}$, un effet inattendu avec ce dernier acaricide.

\section{Avantages comparatifs des différents modes d'application (tableau IV)}

La facilité et rapidité d'emploi dans les conditions d'élevage de la Guadeloupe ont été testées chez des éleveurs.

- L'application en "pour-on" est pratiquement impossible dans la majorité des élevages, où l'attache au piquet des animaux ne limite pas suffisamment leurs mouvements pour permettre de s'approcher d'eux et déposer le produit.

- L'application en "spot-on", qui permet de traiter un animal à une distance de 2 à $3 \mathrm{~m}$ est rapide (20 s par animal) et bien adaptée au mode d'élevage à l'attache. Le coût des produits administrables par ce procédé est cependant élevé, revenant à 6 à $8 \mathrm{~F}$ par bovin et par traitement (4).

- L'application en aspersion est plus longue (45 s), voire davantage $(60 \mathrm{~s})$ si l'éleveur fait lui même le traitement. Le coût en produit pour le traitement d'un bovin va de $0,45 \mathrm{~F}\left(\right.$ Sébacil $\left.^{(B)}\right)$ à $0,80 \mathrm{~F}$ (Taktik $\left.{ }^{\circledR}\right)$ et à $1,1 \mathrm{~F}$ (Butox $50^{(9)}(4)$.

Compte tenu des temps nécessaires de trajet entre élevages ( $n=46, t=5 \mathrm{~min}$ ), de discussions avec l'éleveur ( $n$ $=32, t=2 \min 30 \mathrm{~s})$, de préparation du matériel ( $1 \mathrm{~min} 7 \mathrm{~s}$ en aspersion, $n=38 ; 30$ s en "spot", $n=38$ ), de remplissage de la cuve d'aspersion (estimé à $10 \mathrm{~min}$ tous les 30 éleveurs) et de détiquage, il faut $17 \mathrm{~min} 30 \mathrm{~s}$ pour détiquer un élevage moyen de 8 têtes en aspersion et $10 \mathrm{~min}$ 40 s pour détiquer un cheptel identique en "spot". Ce gain de temps avec ce procédé peut générer des économies très substantielles en matériel (pas de réservoir ni pompe), véhicules (produits prêts à l'emploi) et frais dé personnel lors de campagnes de détiquage organisées et systématiques.

\section{Discussion et Conclusion}

Divers acaricides testés en Guadeloupe sur des animaux élevés au pâturage se sont montrés actifs contre les mouches piqueuses et surtout contre la principale peste du bétail dans les Petites Antilles, la tique Amblyomma variegatum. Parmi les produits en aspersion, l'amitraze (Taktik ${ }^{\text {( ) }}$ a une efficacité de 99 p. 100 sur les tiques adultes. Une efficacité encore meilleure, proche ou égale à 100 p. 100 , a été obtenue avec deux acaricides pyréthrinoïdes prêts à l'emploi, applicables en dépôt dorsal sur la ligne du dos ("pour-on"), ou en taches ("spot-on"): la deltaméthrine en formulation "pour-on" et la fluméthrine (Bayticol "pour-on"()). Le coût de ices produits prêts à l'emploi est élevé, mais leur parfaite efficacité, leur faible toxicité jointe à l'absence de délai d'attente après appli-

Vitesse d'exécution du détiquage en aspersion et du détiquage en "spot-on" dans les conditions de terrain

\section{Nombre d'élevages}

Nombre d'animaux
Durée par bovin en secondes (moyenne et écart-type)

\begin{abstract}
Aspersion (agent du
groupement sanitaire)
\end{abstract}
Aspersion (traitement par l'éleveur)

Application en "spot-on" (agent du goupement sanitaire)
167

$45 \pm 14$
$61 \pm 24$

$$
20 \pm 7
$$


cation, leur facilité et rapidité d'emploi en "spot-on" comparée aux produits en aspersion, justifient leur utilisation dans le cadre de campagnes d'éradication où le résultat attendu impose des produits de qualité. Les conditions d'élevage en Martinique et dans les îles anglophones des Petites Antilles étant très similaires à celles de la Guadeloupe, les recommandations formulées par cette dernière pourraient être transposées dans la plupart des îles infestées de la région.

Outre leur efficacité immédiate, certains acaricides pyréthrinoides, ont une action rémanente de quelques jours, voire de quelques semaines après leur application. Dans des régions comme les Antilles où cohabitent sur le bétail Boophilus microplus, une tique monoxène très prolifique, qui possède une forte capacité à développer des résistance, et Amblyomma variegatum, le recours occasionnel ou à intervalles espacés à des produits rémanents peut générer des résistances de Boophilus à ces produits. II faut donc les réserver à des campagnes de lutte intensive (éradication de la babésiose en Nouvelle Calédonie, éradication des tiques aux Antilles, etc.), où le faible intervalle entre les traitements efface les concentrations décroissantes et sublétales (effet de queue) sur la peau des animaux traités, reconnues comme propices aux apparitions de résistance.

Dans le même ordre d'idées, la technique du "spot-on", identifiée comme très pratique sur des animaux élevés au piquet devra être conduite avec rigueur, sur des animaux attachés courts, pour s'assurer de bien administrer toute la dose sur l'animal.

\section{Remerciements}

Les auteurs remercient l'Unité de Recherches zootechniques de l'INRA Guadeloupe qui a mis à leur disposition les animaux expérimentaux, et tout le personnel du Domaine de Gardel qui a aidé à la réalisation des expérimentations. Ils remercient les Programmes POSEIDOM de Guadeloupe et de Martinique ainsi que les sociétés Roussel-UCLAF et Bayer quil ont apporté leur concours financier à certains des essais.

\section{Bibliographie}

1. ABOTT W.S., 1925. A method of computing the effectiveness of an insecticide. J. Econ. Entomol, 18: 265-267.
2. BARRE N., GARRIS GI., 1990. Biology and ecology of Amblyomma variegatum (Acari: Ixodidae) in the Caribbean: Implications for a regional eradication program. J. Agric. Entomol., 7: 1-9.

3. BARRE N., GARRIS G.I., APRELON R., 1993, Acaricides for eradication of the tick Amblyomma variegatum in the Caribbean. Revue Elev. Méd. vét. Pays trop., 46: 349-354.

4. BARRE N., CAMUS E., FIFI J., FOURGEAUD P., NUMA G., ROSEROSETTE F., BOREL H. Tropical Bont Tick eradication campaign in the French Antilles: current status. N.Y. Acad. Sci, (A paraittre)

5. FAO. 1992. Programme for the eradication of Amblyomma variegatum from the Caribbean. Rome, Italy, FAO, 22 p., 9 annexes. (Report)

6. GARRIS G.I., BOKMA B.H., STRICKLAND R.K., 1989. Evaluation of the eradication programme for Amblyomma variegatum (Acari: Ixodidae) on Puerto Rico. Exp. Appl. Acarol, 6: 67-86.

7. GARRIS G.I., BARRE N., 1991. Acaricide susceptibility of Amblyomma variegatum (Acari: Ixodidae) from Puerto Rico and Guadeloupe. Exp. Appl. Acarol., 12: 171-179.

8. GARRIS G.I., BARRE N., CAMUS E., WILSON D.D., 1993. Progress towards a program for the eradication of Amblyomma variegatum from the Caribbean. Revue Elev. Méd. vét. Pays trop., 46: 359-362.

9. LEMCHE J., PEGRAM R.G., 1987. Control of cattle ticks using flumethrin in central Zimbabwe, Vet. Rec, 121: 110-111.

BARRE N., FARGETTON M., APRELON R., COULIBANDO L. Acaricides for tick control programmes in the Caribbean: results of trials conducted in Guadeloupe. Revue Elev. Méd. vét. Pays trop., 1995, 48 (4): $351-356$

Deltamethrin (Butox $50^{\circ}$ ), a pyrethroid acaricide, amitraz (Taktik ${ }^{\infty}$ ), an amidine, and phoxim (Sebacil ${ }^{*}$ ), an organophosphorus compound, used as spray formulations, as well as two pyrethroids formulated as "pour-on" and used as such or as "spot-on", flumethrin (Bayticot "pour-on") and deltamethrin (a "pour-on" formulation) have been used in Guadeloupe on grazing cattle naturally infested with the adult form of Amblyomma varie. gatum. For acaricides in spray formulation, a higher efficacy is observed with amitraz ( $98.5 \%$ infestation reduction). The efficacy is even greater with the two pyrethroids applied as "pour-on" ( 99.3 \% reduction), or "spot-on" (100\% reduction with flumethrin and deltamethrin). In addition, the "spot-on" technique is better-adapted to tethered livestock, the usual Caribbean breeding method. This technique is 2 to 3 times faster than spraying. Despite the higher price of "pour-on" compounds, the reduced time spent on treatment and, consequently, the reduced cost of labour could make this method competitive, especially within a tick eradication programme.

Key words: Cattle - Tick - Amblyomma - Boophilus - Haematobia - Tickborne diseases - Mite control - Synthetic pyrethrin - Deltamethrin - Amitraz - Phoxim - Animal husbandry - Guadeloupe - Caribbean. 